Diese Resultate entsprechen den Formeln:
I. Nicht getrocknet $\mathrm{CuCl}+3 \mathrm{CuO}+10 \mathrm{HO}$. Getrocknet
$\mathrm{CuCl}+3 \mathrm{CuO}+3 \mathrm{HO}$.
II. Nicht getrocknet $\mathrm{CuCl}+3 \mathrm{CuO}+4 \mathrm{HO}$. Getrocknet
$\mathrm{CuCl}+3 \mathrm{CuO}+3 \mathrm{HO}$.

Auf die Formeln der nicht getrockneten Verbindung ist nach neinem Dafürhalten kein Werth zu legen, da man nach kürzerem oder längerem Trucknen verschiedene Mengen Wasser finden wird; doch ist das Verhältniss von $\mathrm{CuCl}: \mathrm{CuO}=1: 3$ constant. Nach den Analysen von Proust hat man dem Körper allgemein die Formel $\mathrm{CuCl}, 3 \mathrm{CuO}+4 \mathrm{HO}$ gegeben.

Die Bildung des hellblauen Pulvers, aus welchem das Grün durch Auswascben entsteht, aus den blauen Krystallen lässt sich einfach durch die Gleichung ausdrücken:

$$
\begin{gathered}
\mathrm{Cu}_{2} \mathrm{Cl}, \mathrm{NH}_{3}+\mathrm{CuCl}, \mathrm{NH}_{3}+\mathrm{HO}+\mathrm{O}=2\left(\mathrm{CuCl}, \mathrm{NH}_{3}\right) \\
+\mathrm{CuO}+\mathrm{HO} .
\end{gathered}
$$

Die Zersetzung dieses Pulvers durch Waschen mit Wasser in Braunsch ıveiger Grün lässt sich schwerlich durch eine einfache Gleichung bezeichnen.

\title{
XLIV.
}

\section{Ueber das Didym und seine wichtigsten Verbindungen.}

\author{
Von \\ C. Mrarignac.
}

(Ann. de chim. et de phys. 3. Ser. XXXV111, 148.)

Seit der 1841 von Mosander gemachten Entdeckung des Didyms im Cerit ist keine wichtige Arbeit über die Verbindungen dieses Metalls erschienen, und unsre Kenntnisse von demselben beschränken sich fast nur auf die sebr kurzen Angaben, welche der Entdecker selbst gegeben hat. H. Watts*) veröffentlichte

-) Quart. Journ. of the Chem. Soc. of London, II. 140. 
1849 eine Notiz über die Trennung des Cers, Didyms und Lanthans; aber er giebt nur beiläufig die Eigenschaften einiger Didymverbindungen und insbesondere des 0xyds an. In einer zu derselben Zeit veröffentlichten Abhandlung* ${ }^{*}$ über die Atomgewichte dieser Metalle beschäftigte ich mich auch mit ihrer Trennung und ich beschrieb die Krystallform des schwefelsauren Didyms. Die meisten der übrigen Verbindungen wurden jedoch nicht beschrieben.

Ich glaubte, dass es von Interesse sei, auch diese zu untersuchen und besonders vergleichungsweise die entsprechenden Verbindungen des Lanthans, welche wohl besser, aber bei weitem noch nicht hinreichend bekannt sind. Vielleicht ist es möglich, eine Mlethode aufzufinden, mittelst welcher sich diese beiden Metalloxyde besser trennen lassen, als nach der bis jetzt bekannten, die keine quantitative Scheidung gestattet. Ich habe immer zuerst die Didymrerbindungen untersucht, über welche positive Kenntnisse vollständig fehlen und ich hoffe später auch auf die entsprechenden Verbindungen des Lanthans, über welche noch Ungewissheit herrscht, zurückzukommen.

Dieser erste Theil meiner Arbeit umfasst nur einige der wichtigsten Verbindungen. Obwohl ich für diese Untersuchungen neue Hengen der im Cerit enthaltenen Oxyde darstellen musste, so habe ich doch dem, was ich in meiner frühern Abhandlung über diesen Gegenstand gesagt habe, nichts Wichtiges hinzuzufügen. Ich habe es nur für vortheilhafter gefunden, vor der Trennung des schwefelsauren Lanthans und Didyms durch Krystallisation, eine erste, allerdings sehr unvollkommene Scheidung ihrer Oxyde vorzunehmen, indem man sie in einem ziemlich grossen Ueberschuss von Salpetersiuure auflöst und in dieser sauren Lơsung durch Oxalsăure mehrere successive Niederschläge erzeugt. Die ersten Fällungen sind viel mehr ins Rosenrothe gefärbt und reicher an Didym, als die letztern, und die Trennung der schwefelsauren Salze erfolgt viel rascher, wenn eins der Oxyde im Verbältniss zum andern schon in grossem Ueberschuss vorhanden ist.

*) Ann. de chim. et de phys. 3. Ser. XXVIII, 209. Dies. Journ. XLVIII, 406. 
Zunăchst muss ich auf die Bestimmung des Atomgewichts des Didyms zurückkommen und an das Verfahren erinnern, durch welches ich es feststellen $z$ u kőnnen glaubte, nämlich durch Analyse des schwefelsauren Didyms, Ein bestimmtes Gewicht des gut getrockneten Salzes wurde mit der Lösung eines bekannten Gewichts Chlorbaryum versetzt, das sehr wenig mehr betrug, als das zur genauen Zersedzung erforderliche. Der gefällte schwefelsaure Baryt wurde gesammelt, gewaschen, geglüht und gewogen. Die Lósung und die Waschwässer wurden concentrirt und dann durch Schwefelsäure die geringe Menge überschüssigen Baryts gefällt. Aus diesem zweiten sehr kleinen und leicbt auszuwaschenden Niederschlage liess sich das Gewicht des übersclıüssigen Chlorbaryums und demzufolge auch des zur genauen Zersetzung des schwefelsauren Didyms angewendeten berechnen. Ich glaubte auf diese Weise den Febler zu vermeiden oder zu vermindern, welcher dadurch entsteht, dass der erste Niederschlag von schwefelsaurem Baryt, selbst nach langem Auswaschen, immer Didymoxyd zurückhält.

Seit dieser Zeit angestellte Versuche, dieselbe Methode zur Aequivalentbestimmung andrer $0 x y d e$ anzuwenden, führten mich zu so unannehmbaren Resultaten, dass ich mich von der Ungenanigkeit dieser Methode überzeugen musste; die Ursache liegt darin, dass der schwefelsaure Baryt, welcher durch die Füllung eines schwefelsauren Salzes mit Chlorbaryum erhalten wird, selbst bei Ueberschuss des letzlern, eine sehr merkliche Menge des löslichen Sulfats mit fortreisst und trotz des Auswaschens zurückhält.

Ich habe früher die Gegenwart des Didymoxyds in diesem Niederschlage nachgewiesen und erwähnt, dass das Gewicht des erhaltenen schwefelsauren Baryts immer hŏher war, als das aus dem angerwendeten Chlorbaryum berechnete; aber ich dachte nicht, dass es schwefelsaures Didym sein könnte, welches dieses Salz nach dem Kochen der mit überschüssigem Chlorbaryum gemengten Lösung enthielt. Ich konnte dies nachweisen, indem ich den schwefelsauren Baryt nach dem Glühen lange mit Salpetersäure kochte.

Durch die von mir befolgte Methode erhielt ich wohl genau das Gewicht des angewendeten Chlorbaryums, aber nicht des schwefelsauren Didyms, denn von dem Gewicht des letztern 
Salzes hătte die Menge abgerechnet werden müssen, welche vom schwefelsauren Baryt zurückgehalten wurde. Man kōnnte streng genommen in die Berechnung der Resultate eine Correction einführen, welche den Fehler eliminirt, wenn man annimmt, dass der Ueberschuss des schwefelsauren Baryts, verglichen mit dem Gewicht des angewendeten Chlorbaryums, genau das Gewicht des zurückgehaltenen schwefelsauren Didyms repräsentirt. Obwohl nun auch diese Correction nicht absolut genau ist, so versuchte ich doch, sie auf die in meiner ersten Abhandlung mitgetheilten vier Versuche anzuwenden, und es ist $z \mathrm{u}$ bemerken, dass die Resultate dieser Versuche viel besser mit einander stimmen, als es anfangs schien. Ich finde für das Aequivalent des schwefelsauren Didyms*):

$\begin{array}{cc}\text { Frühere Resultate. } & \text { Verbesserte Resultate. } \\ 1210,4 & 1197, \mathbf{4} \\ 1206,9 & 1201,5 \\ 1218,7 & 1201,5 \\ 1219,9 & 1197,0 \\ \text { Mittel } & \frac{1214,0}{1199,4}\end{array}$

Ich musste diese Bestimmungen durch andere Methoden zu controliren suchen.

Das schwefelsaure Didymoxyd konnte auch dazu dienen, indem man nicht die Schwefelsăure, sondern das Didymoxyd bestimmt. 1)ie Bestimmung kann mit ziemlich grosser Genauigkeit ansgeführt werden, wenn man das Oxyd mit oxalsaurem Ammoniak fällt. Das oxalsaure Didymoxyd ist in einer neutralen Flüssigkeit vollkommen unlōslich, lässt sich leicht auswaschen und hält keine Schwefelsăure zurück. Um es in Didymoxyd zu verwandeln, genügt es, dasselbe nach dem Rōsten binreichend stark zu glühen, um das Superoxyd zu zersetzen, welches sich in höherer Temperatur immer bildet. Man kann dies mittelst einer sehr starken Spirituslampe mit doppeltem Luftzuge erreichen; sicherer ist es aber, noch in einem Glühofen zu erhitzen. Uebrigens erkennt man, dass hinreichend hohe Tem-

*) Der nämliche Fehler trifft auch meine Aequivalentbestimmungen des Cers und Lanthans, welche aller Wahrscheinlichkeit nach zu hoch sind. Einige Versuche haben mir bewiesen, dass sie reducirt werden müssen und dass sie sich wahrscheinlich der Zahl 575 nähern, welche yor der Entdeckung Mosander's als Aequivalent des Cers galt. 
peratur angewandt wurde, wenn das Didymoxyd vollkommen weiss ist.

Diese Methode lieferte folgende Resultate:

$\begin{array}{llrl}\text { Sulfat. } & \text { 0xyd. } & \text { p. C. } & \text { Aequivalent des 0xyds. } \\ 2,207 & 1,285=58,22 & 696,8 \\ 3,094 & 1,802=58,24 & 697,4 \\ 3,071 & 1,790=58,29 & 698,7 \\ 3,418 & 1,993=58,31 & 699,3 \\ 2,402 & 1,400=58,29 & 698,6 \\ & \text { Mittel }=\overline{58,27} & 698,2\end{array}$

Das Aequivalent suchte ich ferner durch Analyse des Chlordidyms zu bestimmen; dieses für einige Metalle so bequeme Verfahren bietet hier eine Schwierigkeit dar, welche seine Genauigkeit ein wenig vermindert. Ich habe kein wasserfreies Chlorür darstellen kōnnen, das sich ohne Rückstand in Wasser gelöst hătte. Eine neutrale Lösung verliert durch Verdampfen bis zur Trockne einen selır grossen Theil Salzsāure; das trockne Chlorür lāsst beim Auflōsen in Wasser einen reichlichen, krystallinischen, weissen Rŭckstand, welcher ein Oxychlorür zu sein scheint. Man kann diesen Rückstand allerdings auf einem Filter sammeln, um das Gewicht des analysirten Cblorūrs daraus abzuleiten; aber es ist zweifelhaft, ob man so ein vollkommen genaues Resultat erhält. Nach dem Austrocknen bei $100^{\circ}$ enthält dieser Rückstand noch Wasser, welches in dieser Verbindung während der Schmelzung des Chlorürs nicht existirte und es ist möglich, dass sie beim Erhitzen eine theilweise Zersetzung erleidet. Man kann allerdings die Menge des Oxychlorürs sehr vermindern, indem man das Chlorür entweder in einem Strome Chlorwasserstoffgas trocknet und schmilzt, oder es mit reinem Salmiak gemengt im Platintiegel schmilzt. Trotz dieser Vorsichtsmaassregeln erhielt ich beim Lösen in Wasser immer einen sehr merklichen Rückstand von Oxychlorür.

Zufolge dieses Umstandes habe ich das Gewicht des analysirten Chlorürs nicht in die Berechnung des Aequivalents aufgenommen. Indem ich das Chlor mit salpetersaurem Silber, das überschüssige Silber durch Salzsãure, dann das Didymoxyd mit oxalsaurem Ammoniak fällte, ergab mir die Vergleichung der Gewichte des Chlorsilbers und Didymoxyds das Aequivalent des letztern. Ich erhielt folgende Zahlen: 


$\begin{array}{lccc} & \text { I. } & \text { II. } & \text { III. } \\ \text { Gewicht des geschmolz. Chlordidyms } & \mathbf{6 , 0 2 3} & 2,946 & \mathbf{3 , 5 3 8} \\ \text { Unlüslicher Rückstand geglüht } & \mathbf{0 , 1 3 9} & \mathbf{0 , 0 3 0} & \mathbf{0 , 1 5 1} \\ & \text { I. } & \text { II. } & \text { III. } \\ \text { Analysirtes Chlorür } & \mathbf{5 , 8 8 4} & \mathbf{2 , 9 1 6} & \mathbf{3 , 3 8} \\ \text { Chlorsilber } & \mathbf{1 0 , 0 5 8} & \mathbf{5 , 0 2 9} & \mathbf{5 , 8 4 4} \\ \text { Didymoxyd } & \mathbf{3 , 9 4 6} & \mathbf{1 , 9 6 0} & \mathbf{2 , 2 7 6} \\ \text { Aequivalent des Didymoxyds } & \mathbf{7 0 3 , 5} & \mathbf{6 9 8 , 9} & 698,3\end{array}$

Das Mittel dieser Zatilen würde 700,2 sein; es stimmt ziemlich gut mit der, welche die Analysen des schwefelsauren Salzes ergaben. Daher nahm ich die Zahl 700, welche selur nahe das Aequivalent des Didymoxyds ausdrückt; die Zahl 600 wäre demnach' das Aeqquivalent des Didyms.

Berechnet man nach dieser Zahl das Gewicht des Didyms, so findet man für die Resultate der im Yorstehenden angegebenen Analysen in 100 Chlorür:

\begin{tabular}{lrrrrr} 
& & Ber. & \multicolumn{3}{c}{ Gef. } \\
\cline { 3 - 5 } Chlor & 443,2 & 42,48 & 42,25 & 42,62 & 42,63 \\
Didym & 600,0 & 57,52 & 57,48 & 57,61 & 57,59 \\
\hline 1043,2 & 100,00 & 99,73 & 100,23 & 100,22
\end{tabular}

lch gehe jetzt auf die Eigenschaften des Didyms und einige seiner Verbindungen über.

\section{D $i d y m$.}

Ich stellte dieses Metall dar durch Erhitzen von Kalium mit überschüssigem Didymchlorür in einer an dem einen Ende verschlossenen Porzellanrōhre; das Didymchlorür wurde vorher mit Salmiak geschmolzen.

Die Substanz war melır schlackenartig als geschmölzen, obschon hinreichend starke Hitze angewendet worden wai; durch kaltes Wasser wurden daraus Chlorkalium und Chlordidym aufgelöst; es blieb eine pulvrige, grauliche Substanz zurück, von welcher aus sich fortwährend Wasserstoffblasen entwickelten. Es war leicht, darin ein Gemenge eines grauen metallischen und eines krystallinischen graulich-weissen Pulvers, wahrscheinlich Oxychlorür, zu erkennen. Letzteres liess sich durch Schlämmen nicht beseitigen, so dass eine Reinigung und eine Bestimmung des spec. Gew. des Metallpulvers nicht mōglich war. Wurde es in die Flamme einer Spirituslampe geworl'en, so erzeugle jedes Journ. f. prakt. Chemie. LIX. 7 . 
Metalltheilchen einen lebhaften Funken, denen ähnlich, welche die Verbrennung des Eisens in Sauerstoff begleiten.

In einem dieser Versuche fand ich mitten in dieser pulvrigen Masse zwei kleine Metallkurner, welche wahrscheinlich im Augenblicke der Reaction des Kaliums auf Chlordidym geschmolzen waren; sie zeigten eisengraue Farbe, mit ziemlich lebhaftem Glanz auf den Bruchflächen, den sie aber bald verloren; unter dem Hammer platteten sie sich etwas ab, zerfielen hierauf aber in Stïcke. Eins dieser Stücke, auf Kohle vor dem Löthrohre erhitzt, zeigte keine Schmelzung; nach einiger Zeit war es in eine zerreibliche Oxydmasse verwandelt, ohne dass es ein besonderes Pbänomen der Verbrennung zeigte. An der Luft schienen sich diese Stücke in der Kälte nicht merklich zu verändern; dennoch waren sie am folgenden Tage in eine flockige Oxydmasse verwandelt, vielleicht durch den Einfluss des atmosphärischen Sauerstoffs.

Aus diesem scheint hervorzugehen, dass das Didym im pulverförmigen Zustande kaltes Wasser zersetzen kann, nicht aber im geschmolzenen. In jedem Falle bewirkt der Zusatz einer Säure eine lebhafte Entwicklung von Wasserstoff.

\section{Didymoxyd.}

Es scheint nur ein einziges Oxyd zu geben, welches die Rolle einer Basis spielt, denn das Hyperoxyd, von dem später die Rede sein wird, löst sich in den Säuren nur unter Sauerstoffverlust.

Man erhält dieses Oxyd Jeicht durch Glühen des salpetersauren, oxalsauren. kohlensauren Salzes oder des durch kanstische Alkalien gefällten Hydrats. Es ist nur dann braun, wenn es noch Hyperoxyd enthält. Durch starkes Glühen wird es ganz weiss. Der Wasserstoff ist in der Rothglübhitze ohne Einwirkung auf dasselbe und verāndert seine weisse Farbe gar nicht. Sobald es einmal in 0xyd verwandelt worden ist, oxydirt es sich nicht höher und bräunt sich nicht bei gelindem Glühen an der Luft oder beim Schmelzen mit Salpeter; setzt man aber Salpetersäure hinza und glüht es dann gelind, so nimmt es eine dunkelbraune Farbe an, welche bei stärkerem Glühen wieder verschwindet. 
Es ist eine sehr starke Base, obwohl es in dieser Bezielung noch unter dem Lanthanoxyd steht; selbst nach sebr starkem Glühen löst es sich mit bedentender Temperaturerhöhung und ohne Gasentwicklung in den verdünntesten Säuren leicht auf. Es löst sich eben so in den Salmiaksalzen beim Sieden unter Entwicklung von Ammoniak. Es zieht an der Luft sehr begierig Kohlensäure an. Wasser verwandelt es bei Anwendung von Wärme allmählich in Hydrat; die vollständige Umwandlıng erfolgt indessen nur nach zwei bis drei Tagen.

Das Didymoxydhydrat, aus der Lösung des Chlorürs durch Ammoniak oder Kali gefällt, ist gelatinös und der Thonerde ähnlich, aber mit sehr blass-rosenrother Färbung. Es ist fast unmöglich, dasselbe durch Auswaschen von anhängendem Chlorür gänzlich zu befreien. Beim Austrocknen zieht es sich sehr zusammen und wird röthlich-grau. Nach dem Trocknen zuerst in der Leere, dann bei $100^{\circ}$ verlor es durch Glühen $1 \check{5}$ p. C. an Gewicht. Dasselbe ist somit ein Hydrat mit 1 Aeq. Wasser.

$$
\begin{array}{lrr}
\text { DiO } & \mathbf{7 0 0 , 0} & 86,15 \\
\text { HO } & \mathbf{1 1 2 , 5} & \mathbf{1 3 , 8 5} \\
\cline { 2 - 3 } & \mathbf{8 1 2 , 5} & 100,00
\end{array}
$$

Wäbrend des Trocknens hatte es wahrscheinlich etwas Kohlensãure absorbirt.

Die Didymoxydsalze sind im Allgemeinen rein rosenroth gefärbt, wie das schwefelsaure Salz, oder etwas violett, wie das salpetersaure in concentritter Lösung. Kaustische Alkalien fällen daraus gelatinöses Hydrat; die kohlensauren und doppelkohlensauren Alkalien fälen kohlensaures Didymoxyd, welches im Ueberschuss dieser Reagentien vollständig unlöslich ist. Schwefelammonium schlägt gleichfalls ein wasserhaltiges Produkt nieder. Kohlensaurer Baryt fält das Didymoxyd aus seinen Salzen in der Külte langsam, aber vollständig; eben so oxalsaures Ammoniak in neutraler Lösung; Oxalsãure allein fällt es fast vollständig, wenn die Lösung keinen grossen Ueberschuss an starker Säure enthält. Schwefelsaures Kali, Natron und Ammoniak bilden in concentrirten Lösungen sofort und in verdünnten nach einiger Zeit Niederschläge von schwefelsauren Doppelsalzen, die röthlichweiss gefärbt sind, wenig löslich in Wasser, noch weniger in einem Ueberschuss dieser Reagentien, ohne jedoch darin vollkommen unlöslich zu sein; das schwefelsaure Didymoxyd-Natron 
ist unter diesen dreien am wenigsten löslich. Phosphorsäture und Arsensăure bilden beim Sieden in Säuren wenig lơsliche Niederschläge.

Das Didymoxyd färbt vor dem Löthrohre Borax und Phosphorsalz sehr blass rosenroth, ähnlich wie eine sehr kleine Mlenge Manganoxyd. Kohlensaures Natron wird nicht gefärbt.

\section{Didymhyperoxyd.}

Wenn Didymoxyd nicht sehr stark geglüht worden ist, so zeigt es eine rŏtblich-braune, mehr oder weniger dunkle Farbe. Es ist dann im Zustande des Hyperoxyds, löst sich in Sauerstoffsäuren nur unter Verlust von Sauerstoff, in Salzsäure unter Chlorentwicklung. Uebrigens lōst es sich auch in diesem Zustande in den verdünntesten Säuren, absorbirt an der Luft Koblensãure und treibt, wie das Oxyd, das Ammoniak aus seinen Salzen aus. Durch langes Sieden mit Wasser verwandelt es sich in Hydrat, doch ist die Umwandlung nach einigen Stunden kaum wahrnehmbar.

Man kann es erlıalten durch Rösten des oxalsauren oder kohlensauren Didymoxyds, es lıălt aber dann stets Kohlensãure zurück, indem das kohlensaure Salz eine ziemlich starke Hitze zur vollständigen Zersetzung verlangt. Bequemer ist es durch Glūhen des salpetersauren Salzes darzustellen.

Die Bestimmung des überschüssigen Sauerstoffs durch Glühen ist kein genaues analytisches Verfahren; es giebt immer eine zu grusse Menge Sauerstoff, entweder weil das Hyperoxyd Kohlensăure absorbirt hatte, oder weil es einige Spuren Salpetersãure zurückhieit, denn das salpetersaure Salz verlangt eine ziemlich starke Hitze zur vollständigen Zursetzung. Ich bestimmte diesen überschüssigen Sauerstoff durch Auflösen des Hyperoxyds in einem Gemenge von schwefliger Säure, Salzsäure und Chlorbaryum und Wägung des schwefelsauren Baryts. Die aus diesem Hyperoxyd von verschiedenen Bereitungen erhaltene Sauerstoffmenge betrug $0,32,0,49,0,53,0,51 \mathrm{p}$. C.; ein Mal stieg sie bis zu $0,88 \mathrm{p}$. C., aber in einem nur bei Rothglühhitze geglïhten aus salpetersaurem Salz dargestellten Hyperoxyd, das sicher noch Spuren Salpetersäure enthielt, welche die schweflige Säure oxydiren konnten. Es würrle unnütz sein, für ein Hyperoxyd eine Formel aufzustellen, in welchem der überschüssige Saver- 
stoff so gering ist, und nur 1 Aeq. auf 28 Aeq. Oxyd repräsentirt. Vielleicht ist es ein Gemenge von Oxyd mit einem Hyperoxyd von bestimmter Zusammensetzung. Ich fand kein Mittel, diesen Gegenstand aufzuklären. Das Hyperoxyd wird von verdünnten Säuren so leicht angegriffen, dass es, wenn es mit kohlensauren Salzen gemengt ist, diese sich zuletzt auflösen.

\section{Schwefel-Didym.}

Wenn man Didymoxyd mit kohlensaurem Natron und überschüssigem Schwefel glüht, unŁl die geschmolzene Masse mit Wasser behandelt, so bleibt ein graulich-weisser unlöslicher Rückstand, welcher nach dem Trocknen in der Leere durch Glühen im Wasserstoffstrome nichts an Gewicht verliert. Er löst sich in verdinnter Salzsäure ohne Rückstand auf mit schwacher Entwicklung von Schwefelwasserstoff, aber langsamer, wie es scheint, als Didymoxyd und scheint durch diese Behandlung keineswegs in zwei Theile getrennt zu werden, welche sich nur successiv lösen -wŭrden.

Die Analyse ergab, dass er kein Sulfür, sondern ein Oxysulfür war, aus 1 Aeq. Sulfür und $20 x y d$.

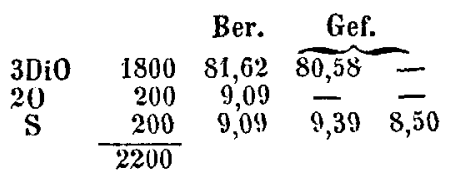

Da das reine Schwefeldidym sich unter diesen Bedingungen nicht bildete, so versuchte ich die Methode, welche Mosander zur. Darstellung-des Schwefellanthans anwendete und nach welcher Fremy die Sulfüre der Erdmetalle erhalten hat. Ich leitete Schrvefelkohlenstoffdampf mittelst eines Wasserstoffstromés über Didymoxyd, das in einer Porzellanrǒhre bis zum Rothglühen erhitzt war. Diese Methode gelang vollständig.

Das so dargestellte Schwefeldidym war nicht geschmolzen; es war pulverförmig, hell bräunlich-grün. Mit Wasser angefeuchtet entwickelt es einen Geruch nach Schwefelwasserstoff, ohne dass sich jedoch Blasen von diesem Gase bilden, wenn man es mit Wasser bedeckt. Die Säuren, selbst sehr verdünnte, zersetzen es unter Entwicklung von Schwefelwasserstoff. Auf Platinblech 
erhitzt kommt es zum Glühen wie Zinnoxydul und verwandelt sich in Oxyd, gemengt mit basischem Sulfat.

Die Zusammensetzung dieses Sulfürs geht aus folgenden Versuchen hervor: 2,742 Didymoxyd, mit 0,392 Sauerstoff, bildeten 3,124 Sulfür. Die 0,392 Sauerstoff wurden somit durch 0,774 Schwefel ersetzt. Die Formel

verlangt $\mathbf{0 , 7 8 4}$.

DiS

\section{Chlor-Didym.}

Die Lösung des Didymoxyds in Salzsäure ist rein rosa gefärbt. Nach hinreichender Concentration durch Erhitzen scheiden sich aus derselben rosafarbene, bisweilen ziemlich grosse hrystalle von wasserhaltigem Chlorür aus. Diese Krystalle sind zerfliesslich, weshalb die genaue Bestimmung ihrer Form schwierig ist. Sie sind rhombische Prismen von $78^{\circ}$, mit einer um $92^{\circ}$ gegen die Prismenflächen geneigten schiefen Basis, und Zuschärfung der stumpfen Winkel der Basis, deren Flächen unter einander einen Winkel von $67^{\circ}$ bilden und demnach um $123{ }^{1 / 2}{ }^{0}$ gegen die Ebene der Basis geneigt sind.

Dieses Chlorür ist in Wasser und Alkohol sehr auflüslich. Seine Lösung kann ohne Entwicklung von Salzsäure vicht bis zur Trockne abgedampft werden; beim Wiederauflösen in Wasser löst sich nur ein Theil des Chlorürs, während ein weisser unlöslicher Rückstand von Oxychlorür bleibt.

Die Analyse des krystallisirten Chlorürs lässt sich approximativ ausfübren, inden man das Wasser durch Erhitzen mil Salmials bestimmt; es bildet sich dann nur eine geringe Menge Oxychlorür.

Die Bestimmung des Chlors durch salpetersaures Silber ist genauer.

$$
\begin{aligned}
& 4,299 \text { verloren } 1,353=31,47 \text { p. C. } \\
& 6,540 \quad " \quad 2,073=31,70 ", \\
& 5,215 \quad " \quad 1,677=32,16 " \\
& \text { Mittel } 31,78 .
\end{aligned}
$$

1,495 Substanz gaben 1,796 Chlorsilber $=0,444$ Chlor und 0,702 Didymoxyd, entsprechend 0,602 Didym.

Diese Resultate führen zu der Formel:

$$
\mathrm{DiCl}+4 \mathrm{HO} \text {. }
$$




\begin{tabular}{lrrr} 
& & Ber. & Gef. \\
Di & 600,0 & 40,18 & 40,25 \\
Ci & 443,3 & 29,68 & 29,70 \\
4H0 450,0 & $\frac{30,14}{1493,3}$ & 31,78 \\
\hline & $\frac{100,00}{1493}$ &
\end{tabular}

Die Analysen des wasserfreien Chlorürs stehen zu Anfange dieser Abhandlung.

Ich versuchte, das unlüsliche 0xychlorür zu analysiren, welches sich durch Verdampfung des Chlorürs bis zur Trockne bildet; doch habe ich keine constanten Resultate erhalten. Nach dem Trocknen in der Leere verliert es kein Wasser bei $100^{\circ}$. Boim Glühen verliert es aber 10 bis 11 p. C. an Gewicht. Bei lange anhaltendem Glühen an der Luft scheint kein Chlor zu entweichen; es färot sich nicht braun. Es wurde in kalter verdünnter Salpetersäure aufgelöst und Chlor und Didymoxyd wurden direct bestimmt. Das Wasser ergab sich aus dem Verlust.

Die Formel

$$
\text { DiCl, } 2 \mathrm{DiO}+3 \mathrm{HO}
$$

stimmt ziemlich nahe mit den gefundenen Resultaten:

Ber. Gef.

$\begin{array}{lrrrrr}\text { DiCl } & 1043,3 & 37,52 & 35,60 & 31,48 & 36,62 \\ 2 \mathrm{Di0} & 1400,0 & \mathbf{5 0 , 3 5} & 53,36 & 55,64 & 52,09 \\ 3 \mathrm{HO} & \mathbf{3 3 7 , 5} & \mathbf{1 2 , 1 3} & \mathbf{1 1 , 0 4} & 12,88 & 11,29\end{array}$

Man sieht, dass die Produkte von verschiedenen Darstellungen nicht genau dieselbe Zusammensetzung zeigen; vielleicht enthalten sie einen Theil des Oxyds als Gemengtheil. Weun dieses Oxychlorür übrigens lange Zeit der Luft ausgesetzt gewesen war, so löste es sich in Säuren unter Aufbrausen, woraus hervorgeht, dass es Koblensäure absorbirt hatte.

\section{Salpetersaures Didymoxyl.}

Dies Salz ist ausserordentlich löslich in Wasser. Eine verdïnnte Lösung desselben ist rein rosa, durch Concentration nimmt sie einen violetten Reflex an. Eine syrupdicke Lösung erstarrt beim Erkalten zu einer krystallinischen Hasse, die aber zu sehr mit Mutterlauge imprägnirt und zu zerfliesslich ist, als dass sich die Menge des Krystallwassers bestimmen liesse.

Es verliert sein Wasser schwer und wird nur wasserfrei, wenn es über $300^{\circ}$ in glühendeń Fluss kount. Erhitzt man 
es so mit grosser Vorsicht, so erleidet es noch keine Zersetzungr und löst sich vollständig in Wasser auf. Es zeigt dann die Zusammenselzung eines wasserfreien neutralen salpetersauren Salzes.

$$
\begin{array}{cccc} 
& & \text { Ber. } & \text { Gef, } \\
\text { Di0 } & 700 & 50,91 & 50,48 \\
\text { NO }_{3} & 675 & \frac{49,09}{2} & - \\
\hline 1375 & 100,00 &
\end{array}
$$

Das wasserfreie Salz lüst sich in Alkohol von 96 p. C. eben so gut als in Wasser; man liann selbst viel Aether zu dieser Lösung hinzusetzen, olıne dass sie sich trübl. Dennoch löst es sich in Aether allein nicht.

Stärker, als bis zum Schmelzen erhitzt, zersetzt sich das Salz unter Entwicklung von Untersalpetersäure; es wird bald teigartjg, bläht sich auf und hinterlässt einen weissen porǒsen und leichten Rückstand, welcher sich später in braunes Hyperoxyd verwandelt.

Wenn man die Substanz mit Wasser behandelt, sobald es sich zu zersetzen angefangen hat, löst sich neutrales salpetersaures Salz und es bleibt ein röthlich-weisser Rückstand. Dieser enthält Salpetersăure ohne salpetrige Säure, wie man sich durch Behandlung desselben mit Schwefelsäure leicht überzeugen kann.

Um es zu analysiren, kann man das Didymoxyd durch Glühen bestimmen, und die Salpetersäure, indem man es kalt in verdünnter Schwefelsäure löst und mit kohlensaurem Baryt zum Kochen erhitzt, bis kein Didymoxyd mehr in Lösung bleibt. Aus dem Baryt in der filtrirten Flüssigkeit lässt sich die Salpetersäure berechnen.

Die erhaltenen Resultate stimmen ziemlich gut mit der Formel : $\mathrm{NO}_{5}, 4 \mathrm{DiO}+5 \mathrm{HO}$.

\begin{tabular}{lcccc} 
& & Ber. & \multicolumn{2}{c}{ Gef. } \\
4DiO & 2800 & 69,65 & 68,75 & 71,20 \\
NO $_{5}$ & 675,0 & 16,72 & 17,69 & 15,91 \\
$5 \mathrm{HO}$ & $\frac{562,5}{402,5}$ & $\frac{13,93}{100,00}$ & - & -
\end{tabular}

Es ist sehr möglich, dass dieses Produkt cin Gemenge eines basischen Salzes mit Didymoxyd oder Didymoxydhydrat ist. Die zwoi angeführten Analysen beziehen sich auf zwei Produkte von verschiedener Darstellung, bei $100^{\circ}$ getrocknet. 


\section{Phosphorsaures Didymoxyd.}

Eine concentrirte Lösung von Phosphorsãure erzengt in concentrirter Lösung von salpetersaurem Didymoxyd sofort keinen Niederscblag; nach ein bis zwei Stunden entsteht aber ein weisses pulvriges Präcipitat. Zusatz einer grossen Menge Wasser bewirkt diese Fällung sofort; beim Sieden der Lösıng bildet er sich schneller. Der grössere Theil des Didymoxyds wird in diese Fällung mit hineingezogen. Der Niederschlag ist unlöslich in Wasser, sehr wenig löslich in verdünnten Säuren, löst sich aber gut in-starken und concentrirten Säıren.

Nach dem Trocknen in der Leere verliert das Salz durch Glüben 7,02 p. G. Wasser. Um die Menge des Didymoxyds und der Phosphorsäure zu bestimmen, schmolz ich das geg!n̆hte und sorofältig gepulverte Phosphat mit kohlensaurem Natron. Die Zersetzung war nicht vollständig. Nach Behandlung des geschmolzenen Produkts mit Wasser wurde das Didymoxyd geglüht und gewogen, dann in concentrirter Salpetersäure gelöst, die Lŏsung bei $100^{\circ}$ bis zur 'Trockne verdampft. Durch mehrmaligen Zusatz von Wasser und dann Eintrocknen blieb endlich mit Wasser unlösliches Phosphat zurück, dessen Gewicht entweder von dem analysirten phosphorsauren Salz, oder rom Didymoxyd abgezogen wurde.

1,327 Grm. geglühtes Salz hinterliessen 0,994 0xyd, wovon ich 0,210 phosphorsaures Salz abzog; 1,117 Grm. Phosphat enthielten somit 0,784 Didymoxyd oder 70,19 p. C.

Daraus geht hervor, dass dies Phosphat ein basisches Salz ist, obschon es aus sehr suuren Flüssigkeiten erhalten worden war.

$$
\begin{array}{lrrc} 
& & \text { Ber. } & \text { Gef. } \\
\text { P() } & 887,5 & 29,71 & - \\
\text { 3Di0 } & \frac{2100,0}{2987,5} & \frac{70,29}{100,00} & 70,19
\end{array}
$$

Das in der Leere getrocknete Phosphat enthielt übrigens 2 Aeq. Wasser:

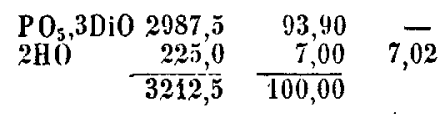

Dieses Wasser verliert es bei $100^{\circ}$ nicht. 


\section{Arsensaures Didymoxyd.}

Arsensäure fällt die Lüsungen der Didymsalze in der Kälte nicht, selbst nach zicmlich langer Zeit. Bringt man die Lösungr aber zum Sieden, so bildet sich ein reichlicher Niederschlag und es bleibt sehr wenig Didymoxyd in Lusung. Das neutrale arsensaure hali bewirlit in Didymsalzen in der Kälte sofort einen Niederschlag. Das arsensaure Salz hat nach diesen beiden Verfahren dargestellt dieselbe Zusammensetzung. Nur, wenn es im Sieden gefällt wird, ist es pulvrig und verändert sich nicht beim Austrocknen. Kalt gefallt ist es etwas gelatinös und nach dem Trocknen etwas durchscheinend und von rosenrother Farbe. In verdünnten Säuren ist es wenig auflöslich.

Nach dem Trocknen in der Leere verliert es bei $100^{\circ}$ kein Wasser; sondern nur beim Glühen; man kann aber die Menge desselben auf diese Weise nicht genau bestimmen. Nachdem das Wasser verjagt ist, zersetzt sich das Salz nach und nach lurch Rösten und verliert fortwährend -an Gewicht. Zur genauen Bestimmung des Wassers genügt es, das Salz mit einem bekannten Gewicht Bieioxyd zu glühen. Uebrigens hat die Analyse keine Schwierigkeit. Das troclne arsensaure Salz wird in Salzsäure gelöst; hierauf kocht man es mit schwefligsaurem Ammoniak zur Umwandlung der Arsensäure in arsenige Säure und fällt das Arsen durch Schwefelwasserstoff. Die filtrirte Lösung wird mit Ammoniak neutralisirt und mit oxalsaurem Ammoniak gefällt. Ich habe nur das Didymoxyd und das Wasser bestimmt. Um sicher zu sein, dass das Oxyd kein Arsenil zurückhielt, löste ich es nach der Wägung wieder in Salzsăure auf und leitete ron Neuem Schwefelwasserstoff hinein.

Die Resultate dieser Analysen führen zu der etwas anormalen Formel: $\quad 2 \mathrm{AsO}_{5}, 5 \mathrm{DiO}, 2 \mathrm{HO}$.

Ich habe sie mit Produkten wiederholt, die auf sehr verschiedene Art dargestellt waren, mit neutralen, mit seht sauren Lősungen, und immer erhielt ich dieselben Zahlen.

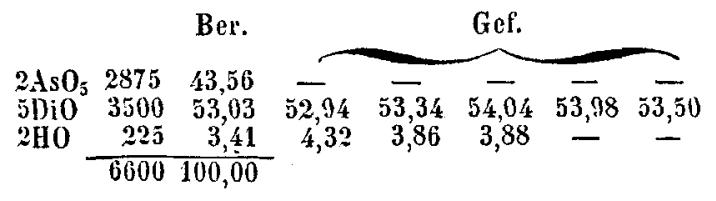




\section{Kohlensaures Didymoxyd.}

Die koblensauren und doppelkohlensauren Alkalien erzeugen in den Didymsalzen sehr voluminǒse Niederschläge von rŏthlichweisser Farbe, vollstāndig unlöslich im Ueberschuss dieser Reagentien. Sie scheinen niemals, selbst nach längerer Berührung mit der Flüssigkeit, worin sie sich bildeten, das perImutterglänzende und blättrige Ansehen der kohlensauren Salze des Cers und Lanthans anzunehmen.

Ich habe ein Salz analysirt, das mittelst doppelkohlensaurem Ammoniak und salpetersatrem Didym in der Kälte bereitet und in der Leere getrocknet war. Das Didymoxyd wurde durch Glühen bestimmt, die Kohlensäure durch Gewichtsverlust, indem man sie in einem gewogenen Apparate durch Schwefelsäure austrieb, das Wasser durch Differenz. Es war ein neutrales kohlensaures $\mathrm{Sal} z$, mit $2 \mathrm{Aer}$. Wasser, $\mathrm{DiO}, \mathrm{CO}_{2}+2 \mathrm{HO}$ :

$$
\begin{array}{rrrc} 
& & \text { Ber. } & \text { Gef. } \\
\text { Di0 } & \mathbf{7 0 0} & \mathbf{5 8 , 3 3} & \mathbf{5 8 , 3 9} \\
\mathbf{C O}_{2} & \mathbf{2 7 5} & \mathbf{2 2 , 9 2} & \mathbf{2 2 , 9 5} \\
\mathbf{2 H 0} & \mathbf{2 2 5} & \mathbf{1 8 , 7 5} & - \\
\hline \mathbf{1 2 0 0} & \frac{1}{\mathbf{1 0 0 , 0 0}} &
\end{array}
$$

Jas Salz verliert bei $100^{\circ} 14,36$ p. C. Wasser, entsprechend 11/2 Aer. Die Analyse des getrockneten Salzes beweist aber, dass sich zugleich etwas Kohlensäure entwickelt.

$$
\begin{array}{lrrc} 
& & \text { Ber. } & \text { Gef. } \\
\text { DiO } & 700,00 & 67,88 & 68,46 \\
\text { CO }_{2} & 275,00 & 26,66 & 25,63 \\
1 / 2 H 0 & 56,25 & 5,46 & - \\
& & \frac{5,400}{100,00} &
\end{array}
$$

Nach diesem schcint das neutrale wasserfreie kohlensaure Salz durch vollständige Austrocknung nicht darstellbar zu sein.

\section{Schwefligsaures Didymoxyd.}

Geglühtes Didymoxyd wird, wenn es in Wasser suspendirt ist, durch einen Strom schwefliger Säure leicht gelöst. Die Lösung ist rein rosenroth. Erhitzt man sie, so trübt sie sich stark, indem sich ein sehr leichter und voluminóser Niederschlag bildet welcher sich durch Erkalten der Flüssigkeit wieder auflost. Wenn man aber die überschüssige schweflige Süure in der Hilze ver- 
jagt, so wird der Niederschlag pulvrig, röthlich-weiss und löst sich nicht mehr durch Erkaltung. Die Lösung bält nur Spuren von Didymoxyd zurück in Folge der Bildung von etwas Scliwefelsäure, denn das schwefligsaure Salz scheint rollständig unIöslich.

Wird es in Wasser suspendirt und mit Chloros behandelt, so löst es sich leicht und verwandelt sich in schwefelsaures Salz; man kann sich leicht überzeugen, indem man die Lösung zur Trockne verdampft, dass reines neutrales Sulfat zurückbleibt. Beim Erhitzen entwickelt es schweflige Säure und verwandelt sich in Oxyd, gemengt mit etwas basischem Sulfat. Nach dem Trocknen in der Leere enthält es 2 Aeq. Wasser, wie das kohlensaure Salz.

1,130 Grm, gaben nach 0xydation durch Clılor in Wasser 1,307 schivefelsauren Baryt und 0,603 Didymoxyd.

$\begin{array}{lrrr} & & \text { Ber. } & \text { Gef. } \\ \text { DiO } & \pi 00 & \mathbf{5 2 , 8 3} & 53,36 \\ \mathrm{SO}_{2} & \mathbf{4 0 0} & \mathbf{3 0 , 1 9} & \mathbf{3 0 , 4 2} \\ 2 \mathrm{HO} & \mathbf{2 2 5} & \mathbf{1 6 , 9 8} & - \\ & \mathbf{1 3 2 5} & \frac{100,00}{100,00} & \end{array}$

\section{Schuefelsaures Didlymoxyd.}

Die Analyse des wasserfreien schwefelsauren Salzes übergebe ich, weil ich sic zu Anfang dieser Abhandlung gegeben habe.

In meiner frühern Abhandlung beschrieb ich speciell die Krystallform des wasserhaltigen Sulfats, welche dem schiefen rhombischen Prisma angehưrt. Ich erwähnte dann, als Anomalie, dass diese Form von der des schwefelsauren Ianthans gänzlich verschieden ist. Dies letztere Salz enthält genau 3 Aecg. Krystallwasser, und ich mahm nach einer approximativen Bestimmung an (deren Abweichung jch irgend einem zufälligen Umstande zuschrieb), dass das nämliche Verhälniss Wasser im schwefelsauren Didym enthalten sei. Seitdem hat mich die Beständigkeit der früher beobachteten Abweichung überzeugt, dass keine vollstündige Analogie zwischen diesen beiden Salzen statfinde und dass die Menge des Wassers im schwefelsauren Didym keiner einfachen Formel entspricht; es enthält $1 / 3$ Aeq. Wasser weniger als das schwefelsaure Lanthan. Dies schien mir ziemlich aussergewōhnlich, so dass ich mich auf alle mögliche Art davon zu 
überzeugen suchte, aber es bleibt mir kein Zreifel. Die Bestimmung des Wassers ist sehr leicht, weil das Salz es unter $200^{\circ}$ vollständig verliert und dann ohne die geringste Veränderung bis zur Rothglühbitze geglüht werden kann. Uebrigens verwittern die Krystalle nicht; sie verlieren in der Leere bei gewöhnlicher Temperatur nicbts an Gewicht. Mehrere Male liess ich das Salz in einer Temperatur krystallisiren, die $\mathbf{1 1}$ bis $12^{0}$ nicht überstieg, um jede Vermengung mit dem Sulfat mit 2 Aeq. Wasser, welches sich in höherer Temperatur bildet, zu vermeiden; ich wählte die Krystalle zur Wasserbestimmung anfs sorgfialtigste aus. Immer erhielt ich dasselbe Resultat.

$$
\begin{aligned}
& 2,025 \text { verloren } 0,410 \text { Wasser }=20,25 \mathrm{p} \text {. C. } \\
& 2,1164 \quad " 0,425 \quad "=20,08 \% \\
& 0,3038,0,0611 "=20,11 " \\
& 6,249 \quad, \quad 1,266 \quad, \quad=20,26 " \\
& 3,893 \quad " 0,799 " \quad=20,53, \\
& 3,847 \quad " 0,776 "=20,17 " \\
& 4,280 \quad, \quad 0,862, \quad=20,14, \\
& 4,256 \quad " 0,853 \quad, \quad=20,04 \text { " } \\
& \begin{array}{lrr} 
& \\
\text { Mittel }=20,20 & \text { p. } & \text { C. } \\
& & \text { Ber. } \\
3\left(\mathrm{SO}_{3}, \mathrm{DiO}\right) & 3600 & 80 \\
8 \mathrm{HO}^{2} & 900 & 20 \\
& 4500 & 100
\end{array}
\end{aligned}
$$

Die Formel

$$
\mathrm{SO}_{3}, \mathrm{DiO}+3 \mathrm{HO}
$$

würde 21,95 p. C. verlangen.

Eine Lüsung dieses Sulfats scheidet in der Wärme und insbesondere boim Sieden einen krystallinischen Niederschlag aus, weicher nur 2 Aeq. Wasser enthält.

2,568 Grm. verloren 0,379 Wasser $=14,76$ p. C.

$$
\begin{array}{lrr}
\mathrm{SO}_{3}, \mathrm{DiO} & 1200 & 84,21 \\
2 \mathrm{HO} & 225 & \frac{15,79}{100,00}
\end{array}
$$

Da die Trennung des Lanthans und Didyms auf den Verschiedenheiten in der Löslichkeit ihrer schwefelsauren Salze beruht, so versuchte ich diese Löslichkeit für das schwefelsaure Didym mit einiger Genauigkeit zu bestimmen. Man erhilt ziemlich abwcichende Resultate, je nachdem man das wasserfreie 
Salz oder eins seiner Hydrate lüst, wie man aus folgender Tabelle urtheilen kann, obwohl die Versuche nicht genau bei denselben Temperaturen ausgeführt wurden waren. Ich begann immer damit, das Salz bis zur Sültigung in kaltem Wasser zu lüsen, dann die Temperatur bei immer höhern Graden stationär zu erhalten und jedes Mal wenigstens 6 bis 8 Stunden lang; dann wor ich eine Portion der Lõsung, verdampfte zur Trockne und glühte den Rückstand bei Dunkelrothglühhitze. Die in der Tabelle angegehenen Zablen bedeuten immer die Henge des wasserfieien schwefelsauren Salzes auf $100 \mathrm{Th}$. Wasser, welches frei oder gebunden in der Lösung sich befindet:

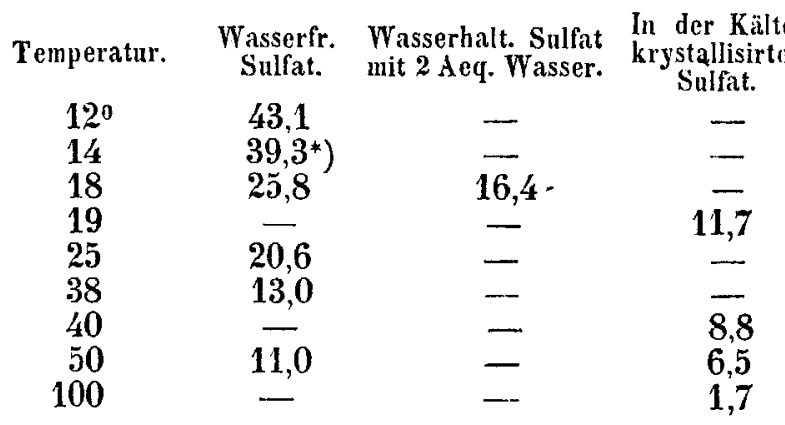

Ich fand überdies, dass das Sulfat mit 2 Aerr. Wasser sein Maximum der Löslichkeit erst nach sehr langer Zeit erreicht. So war die Menge des bei $18^{\circ}$ gelüsten Salzes 13 nach 24 Stunden, und 16,4 nach dem zwerten Tage. Wurde diese Lösung durch Verdampfen in der Leere concentrirt, bis sich der grösste Theil des Salzes in Krystallen ausgeschieden hatte, so enthielt die Mutterlange $34 \mathrm{Th}$. Th. Salz auf 100 Wasser. Dieses Salz scheint also in der Löslichkeit dem wasserfreien gleich zu sein. Uebrigens enthielten die gebildeten Krystalle $8 / 3$ Aeq. Wasser.

Das schwefelsaure Didym ist in Dunkelrothglühhitze beständig, in Weissglühhitze aber zersetzt es sich. Ich konnte seine Zersetzung über der Alkohollampe nicht bewirken. Eine Stunde lang in Iebhaftem Kohlenfeuer geglüht, hatte es 28,03 p. C. seines Gewichts, d. i. 67,28 p. C. oder fast genal $\% / 3$ der ge-

*) Diese Zahl muss zn hoch sein, da die Lösung nur $1 / 2$ Stunde bei dieser Temperatur erhalten warde. 
sammten Menge Schwefelsäure verloren. Man erhält somit das basische $\mathrm{Salz} \mathrm{SO}_{3}$, 3DiO. Es ist ein matt weisses Pulver, vollständig unlöslich in kaltem und heissen Wasser. In verdünnter Salzsäure lüst es sicb nur schwierig, selbst beim Sieden; von concentrirten Säuren wird es leicht aufgelüst.

In einem andern Versuclie, wobei weniger stark geglüht wurde, betrug der Verlust nur 17,5 p. C. Das Produkt theilte sich, mit Wasser behandelt, in lösliches neutrales Sulfat und unlüsliches basisches Sulfat, genau in dem durch diesen Verlust angezeigten Verhälınisse, was die eigenthümliche Existenz dieses dreibasischen Sulfats beweist.

\section{Schwefelsaures Didymoxyd-Ammoniak.}

Mengt man Lösungen von schwefelsaurem Didym und schwefelsaurem Ammoniak, so bildet sich nach kürzerer oder längerer Zeit, je nach dem Grade der Concentration, ein blassrother lirystallinischer Niederschlag. Dieses Doppelsulfat lüst sich in dem 18fachen seines Gewichts Wasser; in einer gesättigten Lösung schwefelsauren Ammoniaks ist es etwas weniger löslich. In Dunkelrothglübhitze verwandelt es sich in schwefelsaures Didymoxyd. Die Bestimmung dieses Sulfats und der ganzen Menge Schwefelsäure lassen folgende Zusammensetzung berechnen:

7,564 Grm. verloren bei $100^{\circ} 0,946$. Wasser $=12,51 \mathrm{p}$. c.

$1,314 "$ liessen nach dem Glühen $0,886=67,43 \mathrm{p}$. C. schwefelsaures Didym mit 28,09 p. C. Schwefelsäure.

1,414 Substanz gaben 1,554 schwefelsauren Baryt $=37,71$ p. C. Schwefelsäure.

$37,71-28,09=9,62$ Schwefelsäure sind im Zustande des schwcfelsauren Ammoniaks; daraus berechnen sich 6,25 Ammoniak.

Man erhält also für die Zusammensetzung dieses Salzes: Sauerst. Verbältn.

\begin{tabular}{|c|c|c|c|c|}
\hline Schwcfels. Didym & $67,43=$ & $\left\{\begin{array}{l}\mathrm{DiO} \\
\mathrm{SO}_{3}\end{array}\right.$ & $\begin{array}{l}39,34 \\
28,09\end{array}$ & $\begin{array}{r}5,62 \\
16,86\end{array}$ \\
\hline Schwefels. Ammoniak & $15,87=$ & $\left\{\begin{array}{l}\mathrm{NH}_{4} \mathrm{O} \\
\mathrm{SO}_{3}\end{array}\right.$ & $\begin{array}{l}6,25 \\
9,62\end{array}$ & $\begin{array}{l}1,92 \\
5,77\end{array}$ \\
\hline Wass & $16,70=$ & $\left\{\begin{array}{l}\mathrm{H} \theta \\
\mathrm{HO} O\end{array}\right.$ & $\begin{array}{r}12,51 \\
4,19\end{array}$ & $\begin{array}{r}11,12 \\
3,73\end{array}$ \\
\hline
\end{tabular}


Diese Resultate führen zur Formel:

welche erfordert:

$$
\mathrm{SO}_{3}, \mathrm{NII}_{4} \mathrm{O}+3\left(\mathrm{SO}_{3}, \mathrm{DiO}\right)+8 \mathrm{HO},
$$

\begin{tabular}{|c|c|c|c|c|}
\hline $3\left(\mathrm{SO}_{3}, \mathrm{DiO}\right)$ & 3600 & 67,61 & $\left\{\begin{array}{l}3 \mathrm{DiO} \\
3 \mathrm{SOO}_{3}\end{array}\right.$ & $\begin{array}{l}39,44 \\
28,17\end{array}$ \\
\hline $\mathrm{SO}_{3}, \mathrm{NH}_{4} \mathrm{O}$ & 825 & 15,49 & $\mathrm{NH}_{4} \mathrm{O}$ & \\
\hline $8 \mathrm{HO}$ & 900 & $\frac{16,90}{100,90}$ & & \\
\hline
\end{tabular}

Das Salz verliert also von den 8 Aeq. Wasser bei $100^{\circ}$ 6 Aeq. -

Ein Produkt anderer Darstellung, für welche ich die beiden Salze in sehr verschiedenen Verhältnissen gemischt hatte, gab mir ähnliche Resultate.

$1,356 \mathrm{Grm}$. gaben bei $100^{\circ} 0,164=12,09$ Wasser.

1,650 " " 1,111 schwefels. Didym $=67,33$ p. C.

Um mich endlich über die Beständigkeit dieses Doppelsalzes zu versichern, wurde eine gewisse Menge davon ausgewaschen, bis sich mehr als die IIälfte gelöst hatte. Der Rest wurde anaJysirt:

1,994 Grm. gaben 1,344 schwefels. Didym oder 67,40 p. C, 1,$906 ; 2,103$ " " Baryt, welchem entspricht :

37,86 p. C. Schwefelsäure.

Die Zusammensetzung war dieselbe geblieben.

\section{Schwefelsaures Dillymoxyd-Natron.}

Vlengt man schwefelsaures Didym und schwefclsaures Natron, so bildet sich augenblicklich ein pulvriger hellrother Niederschlag, welcher zu seiner Lösung ungefälır das 200 [ache seines Gewichts Wasser fordert; in schwefelsaures Natron enthaltendem Wasser ist er noch weniger löslich; vermöge seiner sehr geringen Löslichkeit Jässt sich seine Zusammensetzung- durch Synthese feststellen. Die beiden Salze wurden in dem Verhältniss von 100 schwefelsaurem Didym auf 37 schwefclsaures Natron, d. i. 2 Aer. des erstern auf 1 des zweiten gemengt; die geklärte und decantirte Lösung erzeugte noch einen starken Niederschlag durch Zusatz einer neuen Vlenge schwefelsauren Didyms. Bei 25 schwefelsaurem Nalion auf 100 schwefels. Didym (1 Aerf.: 3 Aeq.) 
gab die geklärte Flüssigkeit keinen merklichen Niederschlag mit einem dieser beiden Salze.

Die Zusammensetzung dieses Doppelsalzes wird daher durch die Formel ausgedrückt:

$$
\mathrm{SO}_{3}, \mathrm{NaO}+3\left(\mathrm{SO}_{3}, \mathrm{DiO}\right)
$$

sie wurde durch folgende Analysen bestätigt:

0,961 Substanz gaben 0,440 Didymoxyd.

1,811

1,895

Didymoxyd.
2,416 schwefelsauren Baryt.

2,492

$" \quad, \quad$ und 0,867

\begin{tabular}{lrrrr} 
& & Berechn. & \multicolumn{2}{c}{ Gefunden. } \\
$3 \mathrm{DiO}$ & 2100 & 46,78 & $\mathbf{4 5 , 7 8}$ & $\mathbf{4 5 , 7 5}$ \\
$\mathrm{NaO}$ & 389 & $\mathbf{8 , 6 7}$ & 4,78 & $\mathbf{4 5 , 1 3}$ \\
$4 \mathrm{SO}_{3}$ & 2000 & $\mathbf{4 4 , 5 5}$ & $\mathbf{4 5 , 7 8}$ &
\end{tabular}

Die Differenz zwischen den berechneten und gefundenen Resultaten rührt ohne Zweifel zum Theil daher, dass der schwefelsaure Baryt schwefelsaures Didym mit niederreisst, was die Bestimmug der Schwefelsăure zur hoch, die des Didymoxyds zu niedrig macht.

Das Salz zu diesen Analysen war geglüht worden; es enthält wirklich Wasser; ich erhielt aber bei der Bestimmung desselben veränderliche Resultáte, von 5 bis $\mathbf{1 0}$ p. C. gewiss deswegen, weil es nicht immer in der nämlichen Temperatur krystallirt war.

\section{Schwefelsaures Didymoxy $b-$ Kali.}

Schwefelsaures Didymoxyd und schwefelsaures Kali geben mit einander gemengt, sofort einen pulvrigen Niederschlag, wenn die Lösungen etwas concentrirt sind und vorzüglich das letztere Salz im Ueberschuss ist; er bildet sich dann langsam fort, wobei er eine mehr körnige und krystallinische Beschaffenheit und röthere Farbe annimmt. Dieses Doppelsalz ist viel löslicher als die entsprechende Natronverbindung; es löst sich in dem 63 fachen seines Gewichts Wasser. Indem ich seine Zusammensetzung synthetisch zu bestimmen suchte, fand ich, dass bei 1 Aeq. schwefelsaurem Kali auf 2 Aeq. schwefelsaures Didym ein Ueberschuss des letzteren in Lösung bleibt. Es ist daher wabrscheinlich, dass das Doppelsalz, welches am meisten geneigt ist sich zu bilden, eine dem Natronsalze ähnliche Formel hat, $\mathrm{SO}_{3}, \mathrm{KO}+3\left(\mathrm{SO}_{3}, \mathrm{DiO}\right)$.

Journ. t. prakt. Chemie. LIX. 7. 
Die mit Produkten verschiedener Bereitungen ausgelührten Analysen gaben keine constanten Resultate. Die zwei Sulfate scheinen sich daher in verschiedenen Verhältnissen verbinden zu kŏnnen.

Ein Doppelsalz, das mit überschüssigem schwefesauren Kali dargestellt war, gab 39 p. C. Didymoxyd. Nachdem es mehrmals mit siedendem Wasser behandelt worden war, gab es 44,96 p. C.

Ein anderes Salz, welches anfangs 42 p. C. Didymoxyd enthielt, gab 44,9 nach dem Sieden mit Wasser.

Die oben angegebene Formel verlangt 44,79 p. C. Didymoxyd. Diese Verbindung scheint also die beständigste zu sein, die einzige wahrscheinlich, welche dem Auswaschen widersteht und besonders einem lang anhaltenden Sieden mil Wasser.

Das nicht geglühte Salz enthält auch Wasser, dessen Menge in den Produkten verschiedener Bereitungen veränderlich war; in den beiden Salzen aber, welche längeres Sieden erlitten halten, betrug es 4,93 und 4,66 p. C., was 2 Aeq. entspricht.

Die Formel

verlangt 4,57 p. C.

$$
\mathrm{SO}_{3}, \mathrm{KO}+3\left(\mathrm{SO}_{3}, \mathrm{DiO}\right)+2 \mathrm{HO}
$$

\section{Oxalsaures Didymoxyd.}

Aus neutralen Lðasungen gefällt ist es pulverig und weiss, etwas rosa; wird es unter Erwärmung in überschūssiger Salpeter- oder Salzsäure gelöst, so scheidet es sich nach dem Erkalten der Lüsung körnig und krystallinisch ab, bisweilen selbst in kleinen rosenrothen Krystallen, von der Form rectangularer Prismen mit einer vierflächigen auf die Kanten des Prismas gestellten Pyramide. Das Salz ist in Wasser vollständig unlöslich, fast unlöslich in Oxalsäure und in sehr verdünnten dlineralsăuren.

An der Luft getrocknet hinterlässt es nach dem Glühen 44 bis 45 Didymoxyd und verliert ungefähr 20 p. C. Wasser bei $100^{\circ}$; aber die Gewichtsverminderung hört nur nach einigen Tagen auf. Das oxalsaure Salz, bei $100^{\circ}$ getrocknet liess $\mathbf{5 5 , 5 3}$ p. C. Didymoxyd.

Daraus kann man schliessen, dass es 4 Aeq. Wasser entbălt, wovon 3 bei $100^{\circ}$ ausgetrieben werden: 
Marignac: Ueber das Didym.

\begin{tabular}{|c|c|c|c|c|c|}
\hline $\begin{array}{l}\mathrm{DiO} \\
\mathrm{C}_{2} \mathrm{O}_{3} \\
4 \mathrm{HO}\end{array}$ & $\begin{array}{l}700 \\
450 \\
450\end{array}$ & $\begin{array}{l}43,75 \\
28,12 \\
28,13\end{array}$ & $\begin{array}{l}\mathrm{DiO} \\
\mathrm{C}_{2} \mathrm{O}_{3} \\
\mathrm{HO}\end{array}$ & $\begin{array}{l}700,0 \\
450,0 \\
112,5\end{array}$ & $\begin{array}{r}55,45 \\
35,64 \\
8,91\end{array}$ \\
\hline & $\overline{1600}$ & 100,00 & & $\overline{12625}$ & $\overline{100,00}$ \\
\hline
\end{tabular}

Während der ganzèn Dauer dieser Untersuchungen beschäftigte mich der Gedanke, dass die untersuchten Verbindungen nicht einfach seien, sondern ein Gemenge von zwei Oxyden, wovon das eine vielleicht Lanthan wäre; ich habe daher meine ganze Aulmerksamkeit auf alle die Umstände gerichtet, welche diese Meinung hätten bestätigen können. Ich begegnete jedoch nur zwei Thatsachen, welche his zu einem gewissen Punkte diese Zweifel unterstützen können. Die eine besteht in der Ueberoxydation des Didymoxyds, welche nur eine kaum merkliche Gewichtszunahme veranlasst; diese Anomalie liesse sich besser durch die Annahme erklären, dass nur eine kleine Menge der Masse beigemengtes Oxyd die Oxydation erleidet.

Die andere Thatsache ist, die ziemlich abweichende Wassermenge, des schwefelsauren Didymoxyds, woraus man auf ein Gemenge von zwei Salzen von verschiedenen Graden der Hydratation schliessen könnte; diese Meinung ist aber für ausgesuchte sehr deutliche und ausgebildete Krystalle schwer anzunebmen. Andererseits habe ich eine grosse Zahl von Versuchen ausgeführt, durch welche ich die Existenz von zwei verschiedenen 0xyden bestimmt nachzuweisen hoffte, wenn sie wirklich in meinem Oxyd enthalten wären, und sie sind alle ohne Resultat geblieben. So habe ich die Reihe der in der Lösung eines Didymsalzes durch Ammoniak nacheinander bewirkten Niederschläge aufmerksam verglichen, ferner der durch partielle Zersetzung in der Wärme erhaltenen unlöslichen Rückstände des salpetersauren Salzes und des Chlorurs, beim Wiederaufnehmen in Wasser, viele Male nach einander, ferner durch aufeinanderfolgende Behandlung der erhaltenen Lösungen nit verdünnten Sätren, jedes Mal in solcher Quantität, dass sie nicht vollständig lösten, sowohl das 0xyd oder Hyperoxyd, oder das Sulfür oder Oxysulfür, und niemals babe ich eine Verschiedenheit in den Eigenschaften der zu Anfang oder zu Ende einer jeden dieser Versuchsreihen erhaltenen Produkte beobachtet. Die Einwirkung des Chlors auf in Wasser suspendirtes Didymoxyd gab ebenfalls kein Resultat. Das Hydrat löst sich leicht, ohne bis zuletzt sein Ansehen zu verändern, das Hyperoxyd löst sich auch, aber 
langsamer. Der Theil welcher sich zuletzt löst, unterscheidet sich in Nichts von dem, welcher zuerst angegriffen wurde.

Trotz der weiter oben angestellten Betrachtungen glaube ich annehmen zu können, dass mein Didymoxyd wohl einige Spuren Lantbanoxyd enthalten konnte, das die Krystallisation des Sulfats nicht vollständig elimirt hatte, dass es wesentlich aber aus einem einzigen Oxyd bestand, dessen Eigenschaften durch jene Beimengung nicht wesentlich verändert wurden.

\title{
XLY.
}

\section{Ueber die Einwirkung des Ammoniaks auf einige arsenigsaure Metalloxyde.}

\author{
Yon \\ Aimé Girard.
}

(Compt. rend. XXXVI, 793.)

Die in Ammoniak löslichen Oxyde sind ebenso in diesem Alkali auflöslich, wenn sie mit arseniger Säure verbunden sind; die Lösungen aber verhalten sich nicht in gleicher Weise.

Das arsenigsaure Kobalt, Nickel, Eisenoxyd lösen sich in Ammoniak, besonders wenn sie frisch gefällt sind, vollständig auf; aber es entsteht dadurch keine Verbindung zwischen dem arsenigsauren Salze und Ammoniak; es ist eine einfache Lósung, welche nach Verlauf einer gewissen Zeit sich zersetzt und vollkommen unverändertes arsenigsaures Salz fallen lässt. Nur das arsenigsaure Silber und Kupfer gaben eine gut bestimmte Reaction.

Das arsenigsaure Silber löst sich, noch bevor es schwer und körnig geworden ist, in Ammoniak auf, und man erhält eine farblose Lösung, welche bei Zusalz von etwas Alkohol nach nach einigen Tagen weisse, durchsichtige Krystalle absetzen lăsst. Diese sind vierseitige, um einen gemeinschaftlichen Mittelpunkt gruppirte Tafeln. Dies Salz ist in Wasser und Alkohol unlosslich, loslich in Ammoniak und Salpetersäure, welche es in arsensaures Salz umwandelt. Salzsäure zersetzt es in Chlorsilber und arse- 